\title{
On when guidelines conflict: Patient safety, quality of life and CAUTI reduction in patients with spinal cord injury
}

\author{
John P. Lavelle ${ }^{1}$
}

Received: 21 May 2019 / Accepted: 22 May 2019

(c) International Spinal Cord Society 2019

Mr. Jones, as you are informed, as his attending physician, has a CAUTI. The news comes by imperious email expecting your comments and action. Mr. Jones is a tetraplegic patient admitted through the ER with a very obvious left leg cellulitis, the most likely cause for his $101^{\circ} \mathrm{F}$ fever. His bladder is managed by an indwelling transurethral catheter, as he had a prior sphincterotomy and was fed up with urinary incontinence. A urine culture for 'dirty' looking urine was ordered by the ER. He does not have any urinary symptoms, or changes in urinary symptoms or signs. The catheter is changed regularly and well maintained with no apparent complications. The ER urine culture is positive for a growth of E. coli and K.pneumoniae with $10^{5} \mathrm{CFU} / \mathrm{ml}$, which are MRDOs. Antibiotic treatment would require IV antibiotics.

This is a hypothetical scenario for illustration purposes. Clinical diagnosis of UTI in SCI patients requires symptoms, this has been the standard in urology and rehabilitation medicine for several years [1-4].

Mr. Jones should not be treated for a UTI, as he has asymptomatic bacteriuria. Treating asymptomatic bacteriuria would lead to more resistant organisms, secondary complications of the antibiotic administration and likely secondary courses of antibiotics in a vain attempt to 'clear' the infection and obtain a sterile urine result, which due to the indwelling catheter, is unlikely to happen.

The problem is that Mr. Jones does not have a clinical UTI. Unfortunately, the NHSN guideline definition [5] for a CAUTI is met and thus the hospital infection control personnel assign a CAUTI to Mr. Jones, as he has a fever and a positive urine culture with an indwelling transurethral catheter. The importance of this administrative assignment

John P. Lavelle

John.lavelle@va.gov

1 Veterans Affairs Palo Alto Health Care System, Urology Section (112), 3801 Miranda Ave, Palo Alto, CA 94304, USA of CAUTI to Mr. Jones, is that it affects the hospital's standing, in terms of HAIs and other surveillance metrics that are used to determine quality-of-care delivery and reimbursement.

Dr. Davis's case series illustrates the fallout from trying to decrease the CAUTI rates in hospital systems. Nursing services have pushed through protocols to remove urinary catheters early, which while laudable, and often appropriate, can, in special circumstances (as described in this case series), be dangerous to the patient with serious repercussions. This is clearly not the intent or the desired outcome of the protocols, which are designed to avoid inappropriate CAUTI assignments.

Interestingly, if all of Dr. Davis's patients had been converted into a suprapubic catheter management, earlier, or had an incontinent ileovesicostomy, then CAUTI could not be applied to his patients, as the definitions would not meet the NHSN guidelines to administratively assign a CAUTI by the hospital infection control. While SP indwelling catheters avoid the ugly- looking traumatic hypospadias in male patients or the disastrous bladder neck erosion in female patients, these patients can and do get symptomatic UTIs (cystitis and pyelonephritis). However, these do not count for CAUTI, which they clearly are. The CDC should consider revision of the CAUTI guideline definitions to allow for these special circumstances.

\section{Compliance with ethical standards}

Conflict of interest The authors declare that they have no conflict of interest.

Publisher's note: Springer Nature remains neutral with regard to jurisdictional claims in published maps and institutional affiliations. 


\section{References}

1. The prevention and management of urinary tract infections among people with spinal cord injuries. National Institute on Disability and Rehabilitation Research Consensus Statement. January 27-29. J Am Paraplegia Soc. 1992;15:194-204.

2. Medicine CFSC. Bladder Management for Adults with Spinal Cord Injury: A clincal practice guideline for health-care providers. 2006. https://pva-cdnendpoint.azureedge.net/prod/libraries/media/pva/ library/publications/cpgbladdermanageme_1ac7b4.pdf.
3. Urological Infections Arnhem, The Netherlands. EAU Guidelines Office, 2019. https://uroweb.org/guideline/urological-infections/.

4. NG 113: Urinary Tract Infection (Catheter Associated): Antimicrobial Prescribing 2018. https://www.nice.org.uk/guidance/ ng113.

5. Network NHS. Urinary TractInfection (Catheter-Associated Urinary Tract Infection [CAUTI] and Non-Catheter-Associated Urinary Tract Infection [UTI]) and Other Urinary System Infection [USI]) Events. 2019. https://www.cdc.gov/nhsn/pdfs/pscmanual/7pscca uticurrent.pdf. 